\title{
Fosbretabulin Disodium
}

National Cancer Institute

\section{Source}

National Cancer Institute. Fosbretabulin Disodium. NCI Thesaurus. Code C2503.

The disodium salt of a water-soluble phosphate derivative of a natural stilbenoid phenol derived from the African bush willow (Combretum caffrum) with potential vascular disrupting and antineoplastic activities. Upon administration, the prodrug fosbretabulin is dephosphorylated to its active metabolite, the microtubule-depolymerizing agent combretastatin A4, which binds to tubulin dimers and prevents microtubule polymerization, resulting in mitotic arrest and apoptosis in endothelial cells. In addition, this agent disrupts the engagement of the endothelial cell-specific junctional molecule vascular endothelial-cadherin (VE-cadherin) and so the activity of the VE-cadherin/betacatenin/Akt signaling pathway, which may result in the inhibition of endothelial cell migration and capillary tube formation. As a result of fosbretabulin's dual mechanism of action, the tumor vasculature collapses, resulting in reduced tumor blood flow and ischemic necrosis of tumor tissue. 